\title{
Reporting on work-related low back pain: data sources, discrepancies and the art of discovering truths
}

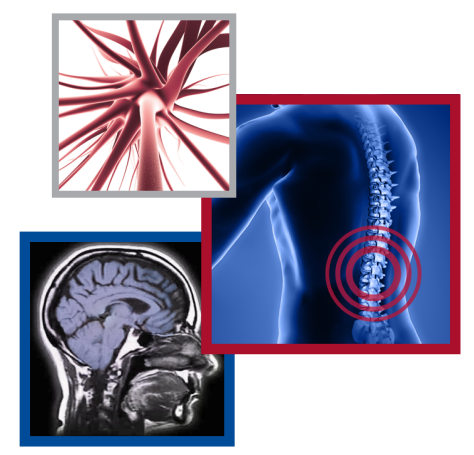

\author{
Xiangning Fan' \& Sebastian Straube $e^{*, 1}$
}

\section{Practice points}

- Work-related back pain is common, and there is significant morbidity associated with this condition. Therefore, it is an important target for preventive efforts.

- Existing data sources may underestimate or overestimate the true population burden of work-related back pain, and this may have important policy and program implications.

- Workers' compensation data are commonly used to estimate the population burden of work-related disease, but estimates derived in this manner are susceptible to reporting bias and under-reporting. Nevertheless, workers' compensation data provide important information about workers' ability to work, disability status and duration, and claims cost, and this information is otherwise inconsistently captured in other data sources, or not at all.

- Population-based surveillance efforts to establish the public health impact of work-related back pain provide a high level overview of the potential burden of work-related back pain, with limited ability to examine specific ergonomic or psychosocial risk factors at the level of the individual workplace. Work-relatedness of cases of back pain identified via population surveillance, and which are attributed to the workplace, is difficult to definitively establish in the absence of a formal adjudication mechanism.

- Attempts to synthesize the existing literature on work-related back pain are hampered by lack of comparability of exposure and outcome measures. Existing attempts at literature synthesis to date have, to our knowledge, not addressed many relevant aspects of work-related back pain.

Work-related pain is unique in the pain context as it is, in theory, tied to one or more workplace activities and is therefore preventable. Back pain is a leading cause of lost workplace productivity, absence from work and reduced quality of life. Aggregate estimates of the workrelated contribution to the overall burden of back pain vary, which may reflect incomplete reporting, inconsistency in data collection and coding between studies and jurisdictions, or, alternatively, genuine differences between occupational groups and countries. It is therefore important for researchers, policy analysts and program development personnel in the fields of pain medicine and occupational medicine to have a thorough understanding of the appropriate use and inherent limitations of the data sources which report on this topic.

First draft submitted: 27 October 2015; Accepted for publication: 24 February 2016; Published online: 15 April 2016 


\section{KEYWORDS}

- data sources - population surveillance $\bullet$ surveillance systems • work-related back pain
The public health impact of work-related back pain

Back pain is a common condition with a substantial impact on work ability. Overall, approximately $15 \%$ of all workers with back pain are estimated to take some time off work, although some studies report estimates in excess of $50 \%$ [1]. Work-related back pain is estimated to account for some 21.8 million disabilityadjusted life years (DALYs), 35\% of all DALYs arising from occupational risk factors globally, and result in a population attributable fraction of $26 \%$ worldwide, albeit with significant variation depending on age, gender and geographical region [2]. This latter estimate for population attributable fraction seems to have been stable over the past decade [3], suggesting that preventive efforts to date have had little efficacy in reducing the population burden over time.

Work-related pain differs from pain of other etiologies in that it is, in principle at least, preventable. The importance of psychological stressors at work notwithstanding, ergonomic factors at the worksite are typically thought to be causal in the development of work-related back pain. A number of ergonomic risk factors thought to be relevant to the workplace have previously been identified [4,5], and there is much interest in the development and evaluation of workplace interventions to reduce the risk of back pain [6-8]. Published guidance for employers on workplace ergonomics programs to prevent work-related back pain and other work-related musculoskeletal disorders $[9,10]$ has been readily available for some time. Nevertheless, work-related back pain, and more generally, work-related musculoskeletal disorders, continue to be a topical issue and contribute substantially to time off work and lost productivity among workers [11].

In the Global Burden of Disease Study 2010 [2], there was a more than twofold difference in DALYs between regions, and a more than threefold difference in the relative risk of back pain by occupational group between the highest- and lowest-risk groups. These differences suggest that occupational factors are associated with, and even possibly causal for, at least some of the population burden of back pain, and that preventive efforts may be able to reduce population morbidity related to this condition. Previous attempts to evaluate factors which may explain the differences in disabling musculoskeletal pain between populations have identified residual differences in reported pain prevalence, even after accounting for reasonably expected confounders, including demographic, physical and psychosocial factors $[12,13]$. There is therefore a compelling public health argument to be made for a comprehensive and in-depth understanding of the reasons for these apparent differences, with the aim of better understanding the underlying phenomenon of work-related back pain, identifying vulnerable subpopulations of workers, and guiding preventive measures.

\section{The nature of the reporting problem on}

\section{work-related back pain}

A variety of work-related outcomes have traditionally been reported in the occupational medicine literature, including incidence and prevalence of occupational injuries and occupational diseases, workplace fatalities, workplace compensation claims, work-related disability and absenteeism from work, among others. However, there are additional important outcomes which are less consistently reported. These include the proportion of workers in the workplace with chronic pain (functional-limiting or otherwise) who are nevertheless still working, and the proportion of workers who take analgesics to treat chronic pain, although such medications may themselves interfere with work ability. Additionally, in the context of this research topic, general 'back pain' is the health outcome often studied, rather than more discrete spinal pathologies such as facet degeneration, sciatica, and spinal stenosis, among others. It is estimated that some $46 \%$ of European workers [11] and approximately $20 \%$ of US workers [14,15] report backache at any given time, although most will not be functionally disabled from work activities. It is additionally unknown what proportion of workers are reliant on pain medications to perform essential job tasks, although it is clear that pain medications, particularly opioids [16], have important workplace implications for workers in safety-sensitive positions and potentially also for those who perform decision-critical, non-safety sensitive work.

Compounding the difficulty of studying workrelated back pain, some workers with back pain will have non-work-related back pain, or back pain that is exacerbated by, but not caused by, work. It is unclear to what extent workplace policies, programs and compensation frameworks for occupational injuries and illnesses account for these latter workers, who are a heterogeneous group. Operational definitions for these latter 
workers are not agreed upon, the natural history of their symptoms is not well elucidated, and they present a causation dilemma within established workplace systems that deal with occupational injury and illness, as their back pain is in part or entirely related to nonwork factors. Workers with back pain are therefore a group about which conclusions are difficult to draw, despite multiple attempts in the research literature to study the phenomenon of work-related back pain, and the development of an extensive literature on this topic.

Aggregate data underpinning important policy and programs on the topic of workrelated back pain are derived from a number of sources, including workers' compensation systems, population-based surveillance efforts and attempts at literature synthesis. An explicit discussion of the strengths and limitations of existing data sources is important when such data are used for policy and program development.

\section{Workers' compensation datasets}

In most North American jurisdictions, workers' compensation systems are the only payers to provide financial benefits for work-related injuries and illnesses for covered workers $[17,18]$. European models of social insurance are similarly comprehensive [19], though there are, of course, differences between jurisdictions. Because of this theoretically wide-ranging and inclusive coverage, workers' compensation datasets (where they exist) are an attractive source of information for researchers, government agencies and others interested in the burden of work-related conditions.

Workers' compensation databases are comprehensive administrative databases which provide population-level data for covered industries and employers in jurisdictions with an 'exclusive remedy' and 'exclusive payer' clause in legislation, although it should be noted that, particularly in the USA, these two conditions exist in only a few state-based systems [20]. It has been previously noted that the primary purpose of workers' compensation databases is for claims administration rather than research; nevertheless, they can be an important data source for public health researchers as data typically collected include the nature of the injury or disorder, the part-ofbody affected, the event or exposure, industry sector, occupation, the worker's ability to continue working and disability status [20]. In fact, because workers' compensation data are collected for claims administration, information on the worker's ability to work, disability status and duration, and claims cost is routinely noted, and these data are otherwise inconsistently captured in other data sources, or not at all [20].

Workers' compensation claims data capture only those workers who make a claim, and claims acceptance generally requires a medical diagnosis of pathology unless presumptive causation has been established in the relevant legislation of a particular jurisdiction. Therefore, while workers' compensation data are inherently specific, this may be at the sacrifice of sensitivity in detecting work-related conditions, as a degree of under-reporting to workers' compensation systems exists [21-27], particularly among temporary, minority, self-employed or illegal workers, who are less likely to make workers' compensation claims and have them accepted [25,28-30]. It should be acknowledged that the problem of under-reporting is widespread and not limited to such groups only [31,32]. Additionally, scant or incomplete information is typically available on industries that are exempt from mandatory workers' compensation legislation, although optional coverage may be available to some employers. Under-reporting within specific workers' compensation systems has been observed to shift substantial healthcare costs to public healthcare systems, leading to underappreciation and underestimation of the true societal burden of work-related conditions. [23,28] Finally, even assuming an insignificant degree of under-reporting to workers' compensation authorities, combining data from multiple jurisdictions is hampered by a lack of similar legislation, agreement as to compensable conditions, and harmonized data standards [20].

\section{Population-based surveillance systems}

The WHO has defined public health surveillance as "the continuous, systematic collection, analysis and interpretation of health-related data needed for the planning, implementation, and evaluation of public health practice" [33]. Within the context of this definition, it is accepted that surveillance efforts should be tied primarily to a public health or policy intervention, although they can be used secondarily for research purposes. Previous large-scale attempts to establish the burden of work-related back pain include the 1988 Occupational Health Supplement to the US National Health Interview Survey [34,35], the triennial Finnish National Work and 
Health Surveys [36], and the European Working Conditions Survey [11]. In the occupational setting, difficulties in obtaining a diagnosis of occupational disease, and gaps in the notification of the proper authorities have previously been described to contribute to the difficulties of performing population surveillance for work-related conditions [37]; the degree to which underdiagnosis and under-reporting are relevant considerations for work-related back pain in the surveillance context is unknown.

Within large population-based surveillance systems, quality data on exposures and outcomes are often limited, and reliance on participant self-report is the rule rather than the exception. In contrast to medical surveillance programs run in individual worksites, which may collect in-depth worker-level information on health status, detailed health information is not typically available on the participants of a population-based surveillance system, due in part to the large size of most surveillance sample sets. No a priori assumptions on the causation of back pain in workers can generally be made when relying upon surveillance data alone, as surveillance data do not require formal medical or legal adjudication of work-relatedness or causative mechanism. For this latter reason, population surveillance is likely to capture a proportion of cases of back pain for which a link with workplace factors is not strong, and may overestimate the extent to which back pain, a common condition in the general population, is related to work. Population-based surveillance efforts on work-related conditions are therefore perhaps best suited to provide a high level overview of the potential burden of work-related back pain, with limited ability at present to examine specific ergonomic or psychosocial risk factors at the level of the individual workplace or worker.

In the surveillance context, as with workers' compensation data, disparate outcome measures are used to describe work-related conditions, limiting the extent to which data can be meaningfully compared across jurisdictions. Reporting of national and international statistics according to both international classification systems (e.g., the International Classification of Diseases [ICD] [38], International Classification of External Causes of Injury [ICECI] [39], Nordic Medico-Statistical Committee Classification of External Causes of Injuries [40]), as well as nation-specific reporting schemes (e.g., US Bureau of Labor Statistics Occupational Injury and Illness Classification System [41], Canadian Standards Association Coding of Work Injury or Disease Information [42], Australian Type of Occurrence Classification System [43]) adds to the difficulty of comparing surveillance data across jurisdictions.

Additionally, surveillance methods for the reporting of work-related injuries can differ from those for the reporting of work-related diseases. It is not always clear whether work-related back pain properly qualifies as a work-related injury or work-related disease, and therefore which surveillance system best captures information in this regard. In the occupational disease arena, there is growing interest in using exposure registries for hazard identification and disease surveillance $[44,45]$, but it has yet to be established whether the use of exposure registries where the exposure is to ergonomic or psychosocial risk factors can yield useful information on workrelated back pain. Of note, the number of workers exposed to ergonomic hazards associated with back pain at work is orders of magnitude higher than the number of workers exposed to more rare exposures (e.g., beryllium) [46] that have previously been of interest in exposure registries.

Notwithstanding the difficulties encountered in surveillance efforts, work-related low back pain has previously been identified as an important 'occupational sentinel health event' [47] and priority for surveillance efforts (e.g., by the Pan American Health Organization) [48].

\section{Literature synthesis}

Given the interest in the overall burden of workrelated back pain, and the availability of individual research studies on this topic, multiple authors have attempted literature syntheses in order to summarize the evidence in this area. Assumptions made during the process of information synthesis are key to the interpretation of the conclusions, but hampered by lack of comparability of exposure and outcome measures across a range of work-related conditions [49], including work-related back pain. To allow for cross-jurisdictional comparisons, occupational categories have previously been used as the basis of comparison [3] in the absence of more specific data on workplace ergonomic risk factors, making the assumption that these risk factors are similarly distributed among occupational groups between jurisdictions. It is not known whether this assumption holds true in general, or how practicable it would be to validate this 
methodology across a range of study types and work-related conditions. There is a need to ensure adequate methodological quality of individual studies and the appropriate use of systematic review and meta-analysis techniques, if meaningful conclusions are to be drawn from such comparisons. Of note, when such literature syntheses are attempted, regional differences in the reported prevalence of work-related low back pain seem to exist even with attempts to evaluate comparable populations $[3,50]$, and further research efforts should focus on the reasons for these apparent differences.

One limitation of many individual studies on work-related back pain is that information on workplace factors is typically limited to occupation or job title. While helpful for epidemiological purposes, characterizing the risk of work-related low back pain by occupational groups, instead of a task-based characterization, is of only limited utility in guiding preventive efforts. The development of job exposure matrices [36] to link ergonomic exposures to specific occupational groups is therefore promising. Job exposure matrices can facilitate attempts at metaanalysis of studies where detailed information on ergonomic risk factors is available for some but not all studies identified in a literature review.

Existing attempts at literature synthesis to date have, to our knowledge, not addressed many relevant aspects of work-related back pain. For example, while individual studies have additionally attempted to clarify exposure-response relationships between workplace exposures and the incidence of low back pain [51,52], few systematic reviews have attempted to answer the same question [53]. Some work outcomes (e.g., disability status) have been acknowledged in previous attempts to define core outcome measures in the study of low back pain $[54,55]$, but have not been universally adopted in the reporting of research study results, and more refinement of work-related outcomes would be of benefit to the research community.

To our knowledge, no dependable consensusbased survey of occupational and pain physicians has been performed to define important outcome measures in observational or intervention studies of work-related painful conditions at large, including back pain, where work-related outcomes, such as time off work and interference with work, should be considered equally as important as pain-related outcomes. A set of core outcome measures for reporting in systematic reviews of intervention studies of painful conditions has been previously suggested [56]. This framework may be usefully adapted for observational and intervention studies on work-related back pain, incorporating outcome measures previously suggested for back pain in general [54,55]. With respect to work-related outcome measures, we suggest that these be considered key: employment status, time off work or absenteeism, attendance at work with pain or presenteeism, interference with work or the need for work modifications due to pain, use of pain medications at work or need for work restrictions, disability compensation and measures of work productivity. Relating to cost, the following outcome measures would seem important: medical care costs (including for imaging and physical therapy), payer (e.g., workers' compensation system vs public or privately funded health insurance) and compensation for lost wages.

\section{Conclusion}

Back pain is a major contributor to morbidity and disease burden in the working population, and an attractive target for surveillance and preventive efforts in the areas of pain medicine and occupational medicine. While estimates of the population burden of work-related back pain can be expected to differ between data sources, some estimate of the expected degree of under-reporting or over-reporting of various data sources, informed by the existing literature, would arguably be of benefit in evaluating the performance of current reporting systems.

\section{Future perspective}

Future advancements in this field could be achieved by establishing robust surveillance networks which collect job or task-specific information on individual workers, harmonizing data collection and reporting standards, and developing a consensus-based set of core outcome measures on which individual research studies would be expected to report and which would form the basis for information synthesis in systematic reviews. This would lay the foundation for crossjurisdictional comparisons, facilitate attempts to study both harmful and protective factors important for work-related back pain, and allow for evaluation of interventions aimed at prevention of this common condition. In addition to a thorough and specific assessment of physical and ergonomic workplace exposures, future research should also include data on important co-factors 
relating to psychological well-being and mental health (e.g., job satisfaction, depression), as these co-factors can have a profound impact on pain perception.

\section{Financial \& competing interests disclosure}

For transparency, S Straube declares honoraria from Oxford Medical Knowledge and an advisory board fee from Daiichi Sankyo, Inc.; all unrelated to this article. Open access publication of this article was made possible by institutional funding by the Department of Medicine, University of Alberta to S Straube. The authors have no other relevant affiliations or financial involvement with any organization or entity with a financial interest in or financial conflict with the subject matter or materials discussed in the manuscript apart from those disclosed.

No writing assistance was utilized in the production of this manuscript.

\section{Open access}

This work is licensed under the Creative Commons Attribution-NonCommercial 4.0 Unported License. To view a copy of this license, visit http://creativecommons.org/ licenses/by-nc-nd/4.0/

\section{References}

Papers of special note have been highlighted as: - of interest; $\bullet \bullet$ of considerable interest

1 Wynne-Jones G, Cowen J, Jordan JL et al. Absence from work and return to work in people with back pain: a systematic review and meta-analysis. Occup. Environ. Med. 71(6), 448-456 (2014).

2 Driscoll T, Jacklyn G, Orchard J et al. The global burden of occupationally related low back pain: estimates from the Global Burden of Disease 2010 study. Ann. Rheum. Dis. 73(6), 975-981 (2014).

- Provides an estimate of the global burden of work-related back pain in the population.

3 Punnett L, Prüss-Ustün A, Nelson DI et al. Estimating the global burden of low back pain attributable to combined occupational exposures. Am. J. Ind. Med. 48(6), 459-469 (2005).

- Paper utilizes a novel approach of using occupational categories as the basis for cross-jurisdictional comparisons. More research is needed to validate this approach.

4 Halpern M. Prevention of low back pain: basic ergonomics in the workplace and the clinic. Baillieres. Clin. Rheumatol. 6(3), 705-730 (1992).

5 Kwon BK, Roffey DM, Bishop PB, Dagenais S, Wai EK. Systematic review: occupational physical activity and low back pain. Occup. Med. (Lond.) 61(8), 541-548 (2011).

6 Maher CG. A systematic review of workplace interventions to prevent low back pain. Aust. J. Physiother. 46(4), 259-269 (2000).

7 Tveito TH, Hysing M, Eriksen HR. Low back pain interventions at the workplace: a systematic literature review. Occup. Med. (Lond). 54(1), 3-13 (2004).
8 Aas RW, Tuntland H, Holte KA et al. Workplace interventions for low-back pain in workers (protocol). Cochrane Database Syst. Rev. 4, CD008159 (2009).

9 Cohen AL, Gjessing CC, Fine LJ et al. A Primer Based on Workplace Evaluations of Musculoskeletal Disorders. National Institute of Occupational Safety and Health, Centers for Disease Control and Prevention, U.S. Department of Health and Human Services, Cincinnati, OH, USA (1997).

10 Fallentin N, Viikari-Juntura E, Waersted M, Kilbom A. Evaluation of physical workload standards and guidelines from a Nordic perspective. Scand. J. Work Environ. Health. 27(Suppl. 2), 1-52 (2001).

11 Eurofound. Fifth European Working Conditions Survey. Publications Office of the European Union, Luxembourg (2012).

12 Coggon D, Ntani G, Palmer KT et al. Disabling musculoskeletal pain in working populations: is it the job, the person, or the culture? Pain 154(6), 856-863 (2013).

13 Straube S, Croft P. Musculoskeletal pain in different occupational groups and different countries. Pain 154(6), 773-774 (2013).

14 Thiese MS, Hegmann KT, Wood EM et al. Low-back pain ratings for lifetime, 1 month period, and point prevalences in a large occupational population. Hum. Factors 56(1), 86-97 (2014).

15 Thiese MS, Hegmann KT, Wood EM et al. Prevalence of low back pain by anatomic location and intensity in an occupational population. BMC Musculoskelet. Disord. 15, 283 (2014).

16 Hegmann KT, Weiss MS, Bowden K et al. ACOEM practice guidelines: opioids and safety-sensitive work. J. Occup. Environ. Med. 56(7), e46-e53 (2014).

17 Reiter JA, Ranta JJ. The Exclusive Remedy Provision: State-by-State Survey. http://apps.americanbar.org
18 Association of Workers' Compensation Boards of Canada. Overview of Canadian Workers' Compensation. What are the Meredith Principles? http://awcbc.org/?page_id=57

19 Ladou J. The European influence on workers' compensation reform in the United States. Environ. Health. 10, 103 (2011).

20 Utterback DF, Meyers AR, Wurzelbacher SJ. Workers' Compensation Insurance: A Primer for Public Health. National Institute of Occupational Safety and Health, Centers for Disease Control and Prevention, U.S. Department of Health and Human Services, Cincinnati, OH, USA (2014).

- This primer is a well-done discussion of workers' compensation data in the USA.

21 Fan ZJ, Bonauto DK, Foley MP, Silverstein BA. Underreporting of work-related injury or illness to workers' compensation: individual and industry factors. J. Occup. Environ. Med. 48(9), 914-922 (2006).

22 Lakdawalla DN, Reville RT, Seabury SA. How does health insurance affect workers' compensation filing? Economic Inquiry 45(2), 286-303 (2007).

23 Thompson A. The consequences of underreporting workers' compensation claims. CMAJ 176(3), 343-344 (2007).

24 Boden LI, Ozonoff A. Capture-recapture estimates of nonfatal workplace injuries and illnesses. Ann. Epidemiol. 18(6), 500-506 (2008).

25 Luckhaupt SE, Calvert GM. Workrelatedness of selected chronic medical conditions and workers' compensation utilization: National Health Interview Survey Occupational Health supplement data. Am. J. Ind. Med. 53(12), 1252-1263 (2010).

26 Centers for Disease Control and Prevention. Proportion of workers who were work-injured and payment by workers' compensation 
systems - 10 states. MMWR Morb. Mortal. Wkly Rep. 59(29), 897-900 (2010).

27 Leigh JP, Marcin JP. Workers' compensation benefits and shifting costs for occupational injury and illness. J. Occup. Environ. Med. 54(4), 445-450 (2012).

28 Lipscomb HJ, Dement JM, Silverstein B, Cameron W, Glazner JE. Who is paying the bills? Health care costs for musculoskeletal back disorders, Washington State Union Carpenters, 1989-2003. J. Occup. Environ. Med. 51(10), 1185-1192 (2009).

29 Premji S, Krause N. Disparities by ethnicity, language, and immigrant status in occupational health experiences among Las Vegas hotel room cleaners. Am. J. Ind. Med. 53(10), 960-975 (2010).

30 American Public Health Association. Workers' compensation reform policy. New Solut. 20(3), 397-404 (2010).

31 Azaroff LS, Levenstein C, Wegman DH. Occupational injury and illness surveillance: conceptual filters explain underreporting. Am. J. Public Health. 92(9), 1421-1429 (2002).

32 Rosenman KD, Gardiner JC, Wang J et al. Why most workers with occupational repetitive trauma do not file for workers' compensation. J. Occup. Environ. Med. 42(1), 25-34 (2000).

33 WHO. Public health surveillance. www.who.int

34 Guo HR, Tanaka S, Cameron LL et al. Back pain among workers in the United States: national estimates and workers at high risk. Am. J. Ind. Med. 28(5), 591-602 (1995)

35 Guo HR, Tanaka S, Halperin WE, Cameron LL. Back pain prevalence in US industry and estimates of lost workdays. Am. J. Public Health 89(7), 1029-1035 (1999).

36 Solovieva S, Pehkonen I, Kausto J et al. Development and validation of a job exposure matrix for physical risk factors in low back pain. PLoS ONE 7(11), e48680 (2012).

-. Describes a job exposure matrix for assigning ergonomic risk factors to occupational groups.
37 Choi BC, Tennassee LM, Eijkemans GJ. Developing regional workplace health and hazard surveillance in the Americas. Rev. Panam. Salud. Publica. 10(6), 376-381 (2001).

38 International Statistical Classification of Diseases and Related Health Problems, $10^{\text {th }}$ Revision (ICD-10). Volume 2 Instruction Manual, 2010 Edition. WHO, Geneva, Switzerland (2010).

39 International Classification of External Causes of Injury (ICECI). WHO, Geneva, Switzerland (2003).

40 NOMESCO Classification of External Causes of Injuries (4th Revised Edition). Nordic Medico-Statistical Committee (NOMESCO), Copenhagen, Denmark (2007).

41 Occupational Injury and Illness Classification Manual, Version 2.01. United States Department of Labor Bureau of Labor Statistics, USA (2012).

42 CSA standard Z795-03 (R2013). Coding of Work Injury or Disease Information. Standards Council of Canada, Canada (2013).

43 Type of Occurrence Classification System, Third Edition. National Occupational Health and Safety Commission, Canberra, Australia (2004).

44 Arrandale VH, Bornstein S, Demers PA. Exposure registries as a tool for epidemiology. Occup. Environ. Med. 74, A49 (2014).

45 Bornstein S, Demers PA, Arrandale VH et al. Tracking Occupational Exposure and Disease: an Analysis of Approaches for the Canadian Context. WorkSafeBC, British Columbia, Canada (2014).

46 Oak Ridge Institute for Science and Education. Beryllium Associated Worker Registry (BAWR). https://orise.orau.gov

47 Rutstein DD, Mullan RJ, Frazier TM et al. Sentinel health events (occupational): a basis for physician recognition and public health surveillance. Am. J. Public Health. 73(9), 1054-1062 (1983).
48 Choi BC, Eijkemans GJ, Tennassee LM. Prioritization of occupational sentinel health events for workplace health and hazard surveillance: the Pan American Health Organization experience. J. Occup. Environ. Med. 43(2), 147-157 (2001).

49 Agius R, Lenderink A, Colosio C. Finding 'new' occupational diseases and trends in 'old' ones. Occup. Med. (Lond.) 65(8), 607-614 (2015).

- Outlines some of the challenges in studying occupational diseases, and describes an attempt to create a pan-European network to address these challenges.

50 Volinn E. The epidemiology of low back pain in the rest of the world. A review of surveys in low- and middle-income countries. Spine (Phila. Pa 1976) 22(15), 1747-1754 (1997).

51 Jansen JP, Burdorf A. Effects of measurement strategy and statistical analysis on doseresponse relations between physical workload and low back pain. Occup. Environ. Med. 60(12), 942-947 (2003).

52 Jansen JP, Morganstern H, Burdorf A. Dose-response relations between occupational exposures to physical and psychosocial factors and the risk of low back pain. Occup. Environ. Med. 61(12), 972-979 (2004).

53 Griffith LE, Shannon HS, Wells RP et al. Individual participant data meta-analysis of mechanical workplace risk factors and low back pain. Am. J. Public. Health. 102(2), 309-318 (2012).

54 Deyo RA, Andersson G, Bombardier C et al. Outcome measures for studying patients with low back pain. Spine. 19(18 Suppl.), 2032S-2036S (1994).

55 Bombardier C. Outcome assessments in the evaluation of treatment of spinal disorders. Summary and general recommendations. Spine 25(24), 3100-3103 (2000).

56 Moore RA, Eccleston C, Derry S et al. "Evidence" in chronic pain - establishing best practice in the reporting of systematic reviews. Pain 150(3), 386-389 (2010).

-. Outlines a set of core outcome measures in pain trials. 